\title{
Polynomials associated with groups of exponent four
}

\section{M.F. Newman, K.W. Weston, and Tah-Zen Yuan}

\author{
Complicated groups of exponent four have been constructed from \\ the ring of polynomials in associating non-commuting \\ indeterminates with coefficients from the field of two elements. \\ The justification of these constructions depends on a \\ computational reduction result. In this note a further reduction \\ is obtained. The expressions involved seem to have an \\ interesting combinatorial structure.
}

The proofs that such groups of exponent four have the claimed properties depend on showing that certain polynomials do not lie in a particular ideal $T$ (described below). This ideal $T$ is one of a set introduced by Bruck [4] in connection with the study of the Burnside questions. In particular $T$ was used by Bachmuth, Mochizuki and Walkup [2] in showing that there are insoluble groups of exponent five. In this note we describe a generating set for the ideal $T$ which makes it easier to work with. Specifically it reduces the amount of computational effort needed to decide whether a given polynomial lies in $T$ or not.

Let $K[X]$ be the ring of polynomials in a set $X$ of associating noncommuting indeterminates with coefficients from a commutative ring $K$ with multiplicative identity 1 . (It is no harder to deal with this case.) If $Y$ is ail ordered finite subset of $X$, the monomial which is the product of the elements of $Y$ in the given order is also denoted $Y$. Let $S(Y)$ denote the sum of the monomials $Z$ where $Z$ rums through all the nonempty sub-ordered-sets of $Y$; so $S(Y)=y+z+y z$ for $Y=(y, z)$.

Received 11 October 1974. 
Let $T(Y)$ be the homogeneous component of degree one in each element of $Y$ of $(S(Y))^{3}$; so $T(Y)=0$ for $Y=(y, z)$ and

$$
T(Y)=x y z+x z y+y x z+y z x+z x y+z y x
$$

for $Y=(x, y, z)$. Let $T$ be the ideal generated by all the $T(Y)$. In the papers quoted a slightly larger ideal is called $T$. There $T$ also contains all polynomials obtained by substituting elements of $X$ for elements of $Y$ in the $T(Y)$. So there, for instance, $2 x x y+2 x y x+2 y x x$ is included. It is easy to see that the results here carry over to that ideal.

The polynomials $T(Y)$ quickly become long sums of monomials as $|Y|$ increases. In characteristic zero $T(Y)$ is the sum of $3^{n}-3 \cdot 2^{n}+3$ monomials where $n=|Y|$. The second column of the table below gives the length of $T(Y)$ in characteristic two for $|Y|$ up to nine. It is, therefore, desirable to find generating sets for $T$ which consist of shorter polynomials. A first result of this kind was obtained in [5] (for the field of two elements); this is stated as Theorem A below. The extent of the improvement can be seen by comparing the second and third columns of the table. The length of the shorter polynomial is $2^{n-2}+4$. The calculations in [1] and [3] use these polynomials. In Theorem $B$ an even shorter generating set for $T$ is given. The fourth column of the table refers to this set; the length of these polynomials is given by

$$
(2+n-2[n / 2]) \cdot 2^{[n / 2]}
$$

where [ ] denotes the integer part of. 
Lengths of polynomials in characteristic two

\begin{tabular}{|r|r|r|r|}
\hline \multirow{2}{*}{$n$} & \multicolumn{1}{|c|}{$T(Y)$} & \multicolumn{2}{|c|}{$C(Z, Y)+C\left(Y^{*}, Z^{*}\right)$} \\
& $|Y|=n$ & \multicolumn{2}{|c|}{$|Y|+|Z|=n$} \\
\cline { 3 - 4 } & & $|Z|=2$ & $|Y| \leq|Z| \leq|Y|+I$ \\
\hline 3 & 6 & 6 & 6 \\
4 & 12 & 8 & 8 \\
5 & 92 & 12 & 12 \\
6 & 302 & 20 & 16 \\
7 & 1312 & 36 & 24 \\
8 & 4294 & 68 & 32 \\
9 & 15110 & 132 & 48 \\
10 & & 260 & 64 \\
11 & & & 96 \\
\hline
\end{tabular}

For $Y, Z$ ordered finite subsets of $X$ let $C(Y, Z)$ be the sum of all the monomials $W Z W^{\prime}$ where $W$ runs through all (including the empty set) sub-ordered-sets of $Y$ and $W^{\prime}$ is the complement of $W$ in $Y$ with order induced from $Y$; thus

$$
C(Y, Z)=x y z+x z y+y z x+z x y
$$

for $Y=(x, y)$. Finally let $Y^{*}$ be the monomial which is the product of the elements of $Y$ in reverse order.

THEOREM A. The ideal $T$ is generated by the polynomials

$$
C(Z, Y)-(-1)^{|Y|} C\left(Y^{*}, Z^{*}\right)
$$

where $Y, Z$ mon through all ordered non-empty finite subsets of $X$ such that $Y, Z$ are disjoint, $|Z|=2$ and $3 \leq|Y|+|Z|$.

THEOREM B. The ideal $T$ is generated by the polynomials

$$
C(Z, Y)-(-1)^{|Y|+|Z|} C\left(Y^{*}, Z^{*}\right)
$$

where $Y, Z$ mun through all ordered finite subsets of $X$ such that $Y, Z$ are disjoint and $|Y| \leq|Z| \leq|Y|+1$.

These theorems are proved together.

LEMMA 1. For every ordered finite subset $y$ of $X$, 


$$
(S(Y))^{3}=\sum T(W)+R
$$

where the sum is taken over all sub-ordered-sets $W$ of $Y$ and $R$ is a sum of monomials each of which has a repeated factor from $X$.

Proof. Let $U(Y)$ be the sum of monomials in $(S(Y))^{3}$ with no repeated factor from $X$. Clearly $\sum T(W)$ is a sub-sum of $U(Y)$. For $y$ in $Y$ the result of putting $y=0$ in $U(Y)-\sum T(W)$ is, inductively, the zero polynomial. Hence $U(Y)-\sum T(W)$ must be a sum of monomials of degree one in each element of $Y$. But $T(Y)$ is the sum of all such monomials. Therefore $U(Y)=\sum T(W)$ as required.

Let $Y$ be an ordered finite subset of $X$ with first element $x$ and let $Z$ be the complement of $x$ in $Y$. Let $B(x, Z)$ be the homogeneous component of degree one in each element of $Y$ of

$$
(S(z))^{2} x+S(z) x S(z)+x(S(z))^{2}
$$

thus $B(x, z)=T(Y)$ for $Y=(x, y, z)$. A proof similar to that of Lemma $I$ yields the following.

LEMMA 2. For $x, z$ as above

$$
(S(z))^{2} x+S(z) x S(Z)+x(S(z))^{2}=\sum B(x, W)+R
$$

where the sum is taken over all sub-ordered-sets $W$ of $Z$ and $R$ is a sum of monomials with a repeated factor.

Let $T(n)$ be the ideal generated by all $T(Y)$ with $|Y| \leq n$ and $R$ the ideal generated by all monomials with a repeated factor.

LEMMA 3. For $|z|=n$,

$$
T(x z) \equiv B(x, z) \text { modulo } T(n) \text {. }
$$

Proof. Work modulo $T(n)+R$. By Lemms 1 , 


$$
\begin{aligned}
T(x z) & \equiv(S(x Z))^{3} \\
& \equiv(x+S(Z)+x S(Z))^{3} \\
& \equiv x(S(Z))^{2}+S(Z) x S(Z)+(S(Z))^{2} x+S(Z) x(S(Z))^{2}+(S(Z))^{2} x S(Z)
\end{aligned}
$$

since $(S(z))^{3}$ belongs to $T(n)+R$. Multiplying this congruence on the right by $S(Z)$ gives

$$
\begin{aligned}
& 0 \equiv s(z) x(S(z))^{2}+(S(z))^{2} x S(z)+(S(z))^{2} x(s(z))^{2}, \\
& 0 \equiv(S(z))^{2} x(S(z))^{2} .
\end{aligned}
$$

Hence $T(x z) \equiv x(S(Z))^{2}+S(Z) x S(Z)+(S(Z))^{2} x$. The result follows from Lemna 2 since $T(x Z)$ is a sum of monomials with no repeated factor.

Observe that for $Z$ non-empty

$$
B(x, z)=C(z, x)+\sum C\left(x, W W^{\prime}\right)-3 C(x, z)
$$

where the sum is taken over all sub-ordered-sets $W$ of $Z$ and $W^{\prime}$ is the complement of $W$ in $Z$. Let

$$
B(Y, Z)=C(Z, Y)-(-1)^{|Y|} \sum C\left(Y^{*}, W W^{\prime}\right)+(-1)^{|Y|} 3 C\left(Y^{*}, Z\right) .
$$

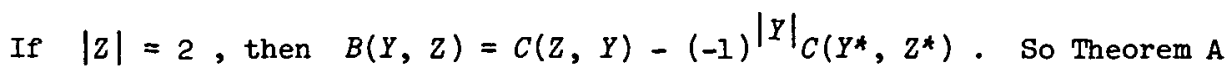
can be proved by establishing a suitable link between values of $B$.

A first step towards this is a link between values of $C$.

LEMMA 4. If $x$ is an element of an ordered finite subset $x_{0}$ of $X$ and $Y$ is the ordered set of predecessors of $x$ in $X_{0}$ and $Z$ is the set of successors of $x$ in $X_{0}$, then

$$
C(x Z, Y)=C(Z, Y x)+x C(Z, Y) .
$$

Proof. From the definition $C(x Z, Y)=\sum W W^{\prime}$. where the sum is taken over all sub-ordered sets $W$ of $x Z$. Hence

$$
\begin{aligned}
C(x Z, Y) & =\sum_{x \in W} W Y W^{\prime}+\sum_{x \in W^{\prime}} W Y W^{\prime} \\
& =x \sum V Y V^{\prime}+\sum V(Y x) V^{\prime}
\end{aligned}
$$


where both sums are taken over all sub-ordered-sets $V$ of $Z$. The result follows.

LEMMA 5. With the notation as in Lemma 4 and $Y, Z$ non-empty

$$
B(Y, x Z) \equiv B(Y x, Z) \text { moduzo } T(|Y|+|Z|) \text {. }
$$

Proof. From the definition

$$
B(Y, x Z)=C(x Z, Y)+(-1)^{|Y|}\left(3 C\left(Y^{*}, x Z\right)-\left[C\left(Y^{*}, W W^{\prime}\right)\right)\right.
$$

where the sum is taken over all sub-ordered-sets $W$ of $x Z$. Hence

$$
B(Y, x Z)=C(x Z, Y)+(-1)^{|Y|}\left(3 C\left(Y^{*}, x Z\right)-\sum C\left(Y^{*}, x V^{\prime}\right)-\sum C\left(Y^{*}, V x V^{\prime}\right)\right)
$$

where both sums are taken over all sub-ordered-sets $V$ of $Z$. Now $B(x, z)=\sum\left(x V V^{\prime}+V x V^{\prime}+V V^{\prime} x\right)-3 x Z-3 Z x$ lies in $T(|y|+|z|)$ so

$$
B(Y, x Z) \equiv C(x Z, Y)+(-1)^{|Y|}\left[\sum C\left(Y^{*}, V V^{\prime} x\right)-3 C\left(Y^{*}, Z x\right)\right)
$$

since $C$ is linear in the second place. Using Lema 4 gives

$$
\begin{aligned}
B(Y, x Z) \equiv & C(Z, Y x)+(-1)|Y|\left(\left[C\left(x Y^{*}, V V^{\prime}\right)-3 C\left(x Y^{*}, Z\right)\right)\right. \\
& +x C(Z, Y)+(-1)|Y|\left(-x\left[C\left(Y^{*}, V V^{\prime}\right)+3 x C\left(Y^{*}, Z\right)\right)\right. \\
& \equiv B(Y x, Z)+x B(Y, Z) \\
& \equiv B(Y x, Z)
\end{aligned}
$$

as required.

Let $n$ be an integer, at least 2 , and $Y, Z$ disjoint subsets of $X$ with $|y|=n-1$ and $|z|=2$, then

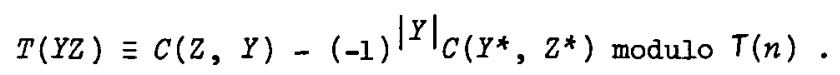

A simple induction over $n$ completes the proof of Theorem A.

In the notation of Lemma 4, and using Lemma 4,

$$
\begin{aligned}
C(Z, Y x)-(-1)^{|Y|+|Z|+1} C\left(x Y^{*}, Z^{*}\right)= & C(x Z, Y)-(-1)^{|Y|+|Z|+1} C\left(Y^{*}, Z^{*} x\right) \\
& -x\left(C(Z, Y)-(-1)^{|Y|+|Z|} C\left(Y^{*}, Z^{*}\right)\right) .
\end{aligned}
$$

Another simple induction over $n$ and repeated use of this yield Theorem $B$. 


\section{References}

[1] Seymour Bachmuth and Horace Y. Mochizuki, "A criterion for nonsolvability of exponent 4 groups", Comm. Pure Appl. Math. 26 (1973), 601-608.

[2] S. Bachmuth, H.Y. Mochizuki and D.W. Walkup, "Construction of a nonsolvable group of exponent 5 ", Word Probzems. Decision problems and the Burnside problem in group theory, 39-66 (Studies in Logic and the Foundations of Mathematics, 71. North-Holland, Amsterdam, London, 1973).

[3] S. Bachmuth, H.Y. Mochizuki and K. Weston, "A group of exponent 4 with derived length at least 4 ", Proc. Amer. Math. Soc. 39 (1973:), 228-234.

[4] R.H. Bruck, Engel conditions in groups and related questions (Lecture Notes. Third Summer Research Institute of the Austral. Math. Soc., Canberra, 1963).

[5] Tah-Zen Yuan, "On the solvability of the freest group of exponent 4 ", (Dissertation, University of Notre Dame, Notre Dame, Indiana, 1969).

Department of Mathematics, Institute of Advanced Studies, Australian National University, Canberra, ACT;

Department of Mathematics,

University of Wisconsin-Parkside,

Kenosha,

Wisconsin, USA;

Metropolitan Mil waukee Association of Commerce,

Mi I waukee,

Wiscons in, USA. 